\title{
Flow periodicity and convection modes in rotating Rayleigh-Bénard convection at low Rayleigh numbers
}

\author{
VISHNU VENUGOPAL T and ARNAB KUMAR DE* \\ Department of Mechanical Engineering, Indian Institute of Technology Guwahati, Guwahati 781039, India \\ e-mail: akd@iitg.ac.in
}

MS received 14 July 2017; revised 11 March 2018; accepted 21 July 2018; published online 19 January 2019

\begin{abstract}
A three-dimensional numerical study on rotating Rayleigh-Bénard convection of water in a cylindrical container with a specific aspect ratio is performed in the present work. The simulations are carried out at four different Rayleigh numbers $\left(3 \times 10^{4}, 5 \times 10^{4}, 7 \times 10^{4}\right.$ and $\left.10^{5}\right)$ and a fixed Prandtl number $(P r=7)$ for a range of rotation rates. Flow structures and their evolution with the addition of rotation to the system are studied in detail. Emphasis is given on the analysis of wall mode and bulk mode convection that appear at different rotation rates. The changes in heat transfer and stability of the system are also investigated. Heat transfer rate is measured by calculating the average Nusselt number at the hot wall. The results show that rotation primarily has an inhibiting effect on heat transfer. For $R a \leq 7 \times 10^{4}$ the decrease in heat transfer is negligible at lower rotation rates, while it declines steeply for higher rotation rates. At $R a=10^{5}$ a small increase in Nusselt number is obtained at low rotation rates before it drops at higher rotation rates. Numerous probes placed at different points within the flow domain are used to investigate the flow regimes and convection modes. The flow initially remains steady at low rotation rates and transforms to a periodic stage with bulk-modedominated convection at moderate rotation rates. Further increase in rotation gives a wall mode convection accompanied by a drastic drop in heat transfer rate before finally approaching a static conductive stage. The dual role of rotation on the stability of Rayleigh-Bénard convection is clearly identified in the present study. At moderate rotation rates, the rotation force destabilizes the system to reach a periodic flow whereas extremely large rotation rate stabilizes it.
\end{abstract}

Keywords. Rayleigh-Bénard convection; wall modes; bulk modes; convection rolls; periodic flows; rotation.

\section{Introduction}

Rayleigh-Bénard convection (RBC) is a bottom heated top cooled configuration of a fluid layer confined between two horizontal surfaces. It is one of the classical problems in fluid dynamics. Rayleigh-Bénard instability occurs when a fluid layer is subjected to an adverse vertical temperature gradient. Due to thermal expansion, the fluid at the bottom (hot side) becomes lighter than the fluid at the top (cooler side). This top-heavy bottom-light configuration is potentially unsteady, and the fluid has a natural tendency to redistribute itself to counter this destabilizing effect. However, the viscosity of the fluid acts as a stabilizing force against this redistribution. For small temperature differences across the plates, no convective flow occurs and heat transfer is only by conduction. With the increase in temperature difference, the destabilizing buoyancy force exceeds the stabilizing viscous force and convection sets in,

*For correspondence followed by an increase in heat transfer rate. Convection rolls appear and regular flow patterns can be observed.

Rotating convection is ubiquitous in atmospheric and geophysical flows. Convection inside earth's crust, oceanic movements and stellar convections are some examples. Further, it finds applications in various engineering fields like cooling of electronic equipment, metal solidification processes, fluid storage, crystal growth and materials processing. Rotating Rayleigh-Bénard convection (RRBC) combines the effects of thermal buoyancy and rotation-induced Coriolis and centrifugal forces. RRBC was first studied by Chandrasekhar [1] in 1953. In his book [2], Chandrasekhar extends Rayleigh's analysis on $\mathrm{RBC}$ to the rotating case. He examined the stability of a fluid layer heated from below, subjected to gravity and Coriolis force. It was shown that the effect of the Coriolis force was to inhibit the onset of convection. The extent of the inhibition depends on certain non-dimensional parameters. From linear stability theory he found out that for any $\operatorname{Pr}$ greater than one, the critical Rayleigh number depends only on the Taylor number $\left(T a=\left(\left(2 \Omega H^{2}\right) / v\right)^{2}\right)$. However, for lower Prandtl number, $R a_{c}$ depends on both 
$\mathrm{Pr}$ and $\mathrm{Ta}$. This was later experimentally confirmed by Fultz and Nakagawa [3]. Chandrasekhar [2] analysed an infinite fluid layer rotating at a constant rate. He found out that for non-rotational case, viscosity has a stabilizing effect on the fluid layer, but when rotation is introduced, viscosity plays a destabilizing role. Chandrasekhar explained this using the Taylor-Proudman theorem [4, 5]. Veronis [6] also observed that rotation has stabilizing effect on convection system. For a range of values of $P r$ and $T a$, instability occurs as a timeperiodic phenomenon called over-stability. He observed that over-stability cannot occur when $P r$ is higher than certain critical values and the rotation rates are sufficiently lower. The effect of aspect ratio on the onset of convection in RRBC was studied by Buell and Catton [7]. It was observed that at large enough rotation rates, lateral walls show a stabilizing effect and the onset of convection is delayed.

Ecke et al [8] identified that the onset of $\mathrm{RBC}$ in rotating cylindrical containers is a Hopf bifurcation, which gives rise to rotating (travelling) waves in the azimuthal direction. It was observed that the frequency of these travelling waves increases with the increase in rotation rates. Goldstein et al [9] investigated the linear stability problem for the onset of convection inside a uniformly rotating cylinder and identified different modes for the onset of convection. They also evaluated the aspect ratio dependence for the onset of these modes. Some of the other prominent theoretical studies in this direction include the works by Herrmann and Busse [10], Kuo and Cross [11], Goldstein et al [12], Zhang et al [13], etc. Zhang and Liao [14] conducted numerical and analytical studies on instabilities in rotating convection for different Prandtl numbers. They identified the instabilities as convection-driven inertial waves and wall-localized travelling waves for small (and moderate) and large Prandtl numbers, respectively. Balasubramanian and Ecke [15] observed a periodic regime at certain rotation rates in their experimental studies on RRBC. The experiments were performed in a square cavity over a range of Rayleigh numbers $10^{7} \leq R a \leq 2 \times 10^{8}$. The occurrence of wall modes and bulk modes at different rotation rates was also visualized. Similar oscillatory convection was observed by Rossby [16] in his experiments with water. It was observed that at any $R a>10^{4}$ an increase in Nusselt number $(\mathrm{Nu})$ of about $10 \%$ can be expected with the increase in rotation rates. The percentage increase in $\mathrm{Nu}$ is also dependent on $\mathrm{Ra}$. It was observed that the $\mathrm{Ta}$ corresponding to maximum heat transfer is an increasing function of $R a$. A detailed study on wall modes, bulk modes and their bifurcations was carried out by Zhong et al [17] and Ning and Ecke [18]. Experiments were performed with water in a cylindrical container with aspect ratios $(\Gamma=D / H) 20$ and 5. Fixing the rotation rates, the temperature difference across the plates was varied to analyse different convection modes and their bifurcations. They observed that based on the rotation rate, the sequence of appearance of the wall and bulk modes varies. At lower rotation rates the bulk convection sets in first and the wall convection begins later, but for higher rotation rate wall modes are the primary bifurcation modes (bulk modes are secondary). A detailed study on the multiplicity of wall modes and their instabilities was performed by Marques and Lopez [19]. It was found that the wall and the bulk modes are spatially separated and tend to interact only in the narrow annular region between them. As the aspect ratio increases the separation becomes more pronounced and the wall modes get more confined to the sidewalls. Wall modes are a result of interaction of the Coriolis force with the lateral walls.

Most of the existing literature on wall and bulk mode convections in RRBC are focussed on identifying the critical Rayleigh number for the onset of these modes at constant rotation rates. In the present study, we keep the $R a$ constant (four cases) and investigate the effect of rotation by decreasing the Rossby number $(R o)$ and identify the appearance of wall and bulk mode convections at specific Ro. We study the bulk to wall mode transition with the increase in rotation rate and connect it with the associated heat transfer dynamics.

The effect of rotation in RBC was studied by Kunnen et al [20] in their numerical simulations at $R a=2.5 \times$ $10^{6}$ and $\operatorname{Pr}=1.0$ for a range of rotation rates. A steep fall in heat transfer rate was observed at high rotation rates though a minor increment of $N u$ up to $5 \%$ was observed at low rotation rates. Similar observations were made by Stevens et al [21] in their numerical studies. It was found that at a fixed Rossby number there exists a $P r$ for maximum heat transfer enhancement. This increase in heat transfer can be explained by the Ekman pumping [22]. Zhong et al [23] investigated the effect of $R a$, $P r$ and $R o$ on heat transfer in turbulent RRBC. A detailed analysis of the flow structures in turbulent RRBC was conducted by Kunnen et al [24, 25]. At very high rotation rates the stabilizing effect of rotation was clearly demonstrated.

The focus of the present study is to observe the effect of rotation at a low Rayleigh number regime with an emphasis on the analysis of periodic flows. In particular, we discuss the appearance of wall mode and bulk mode convection, their transitions, heat transfer rates, stability of the system and flow structures with the increase in rotation rate. The next section discusses the overall solution methodology with the governing equations used, boundary conditions applied followed by a brief note on the numerical techniques employed. The details of the grid independence study and preliminary code validations are also explained in section 2. Section 3 reports the results obtained in the current study, which is followed by principal findings summarized in section 4 . 


\section{Solution methodology}

The problem deals with flow investigation of an incompressible Newtonian fluid confined in a cylinder of diameter $D(2 R)$ and height $H$, with bottom heated $\left(T_{H}\right)$ and top cooled $\left(T_{C}\right)$ configuration. The cylinder is rotated about its vertical axis. A schematic diagram of the flow domain with boundary conditions used is shown in figure 1. The mathematical model of the problem consists of the mass, momentum and energy conservation equations written in the rotating frame of reference using the Boussinesq approximation employing $H$ and $V(=\sqrt{g \beta \Delta T H})$ as the length and velocity scales, respectively:

$$
\begin{aligned}
& \frac{\partial \boldsymbol{U}_{\boldsymbol{R}}}{\partial t}+\boldsymbol{U}_{\boldsymbol{R}} \cdot \nabla \boldsymbol{U}_{\boldsymbol{R}}=-\nabla P+\sqrt{\frac{P r}{R a}} \nabla^{2} \boldsymbol{U}_{\boldsymbol{R}}+\theta \hat{k} \\
& -\frac{1}{R o}\left(\hat{k} \times U_{\boldsymbol{R}}\right) \\
& \nabla \cdot \boldsymbol{U}_{\boldsymbol{R}}=0 \\
& \frac{\partial \theta}{\partial t}+\boldsymbol{U}_{\boldsymbol{R}} \cdot \nabla \theta=\frac{1}{\sqrt{\operatorname{PrRa}}} \nabla^{2} \theta
\end{aligned}
$$

where $\boldsymbol{U}_{\boldsymbol{R}}$ is the velocity vector in the rotating frame, $\hat{k}$ is a unit vector in the vertical direction, $\theta\left(=\left(T-T_{\circ}\right) / \Delta T\right)$ is non dimensional temperature and $P$ is modified pressure incorporating the hydrostatic and centrifugal effects. $R a=g \beta \Delta T H^{3} / v \alpha, P r=v / \alpha$ and $R o=V / 2 \Omega H$ are the Rayleigh number, Prandtl number and Rossby number, respectively, where $\Delta T\left(=T_{H}-T_{C}\right)$ is the temperature difference across the hot and cold surfaces and $T_{\circ}$ $\left(=\left(T_{H}+T_{C}\right) / 2\right)$ is their mean temperature. These coupled equations are solved in the cylindrical domain using finitevolume formulation with collocated arrangement of variables. The convection term is approximated using the 2ndorder Adams-Bashforth scheme while the buoyancy and diffusion terms are by the Crank-Nicholson scheme. In

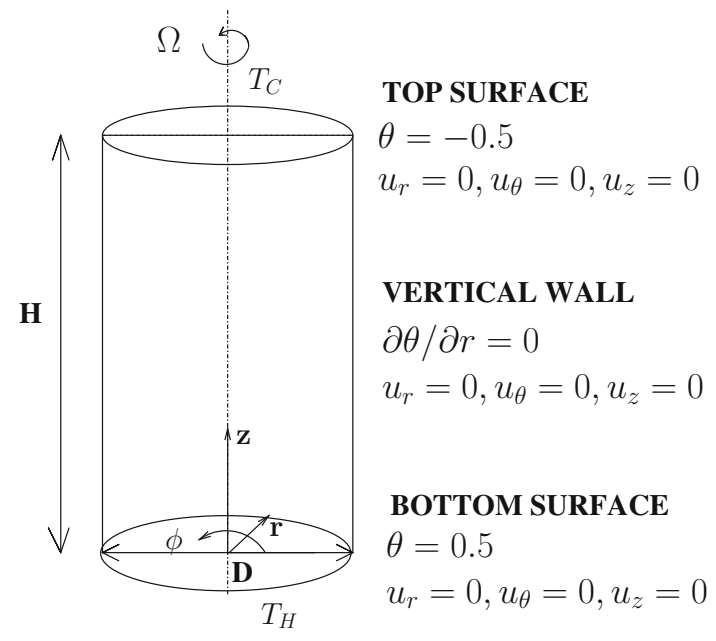

Figure 1. Schematic diagram of the flow domain with relevant boundary conditions. order to adequately address the issue of velocity-pressure coupling, a predictor-corrector-based momentum interpolation method [26] is used as the solution technique. A BiConjugate Gradient Stabilized (BiCGSTAB) technique preconditioned by the Stone's Strongly implicit procedure (SIP) [27] is used to solve all the resulting sparse linear systems. The numerical code is parallelized on an MPI platform and is run on 12 computing processors. For details of the numerical procedures the reference Peter and De [26] can be followed.

\subsection{Comparison with previous literature}

The numerical code is validated for RRBC at low Rayleigh numbers. Simulations are performed in a cylindrical container at $\mathrm{Pr}=6.8, \mathrm{Ro}=0.00245$ for a Rayleigh number range $3600 \leq R a \leq 52000$. The results are compared to those obtained by Rüdiger and Knobloch [28]. Figure 2 shows the mid-plane temperature deviation $\theta^{\prime}\left(=\theta-\theta_{\text {con }}\right)$ for different Rayleigh numbers that are closest to the reported ones, where $\theta_{\text {con }}$ is the linear conduction temperature profile. Dark (light) regions indicate $\theta^{\prime}<0\left(\theta^{\prime}>0\right)$ representing cold descending (warm ascending) fluid. Further, for quantitative assessment of the numerical code, area-averaged Nusselt number at the bottom plate $\langle N u\rangle$ is calculated and compared to that obtained by Oresta et al [29]. Table 1 shows that the results obtained are well within acceptable error limits. The simulations are performed at a fixed Prandtl number $\mathrm{Pr}=0.7$ in a cylindrical container with aspect ratio $\Gamma=0.5$ for a range of Rayleigh numbers. As observed by Oresta et al [29], we obtained a critical Rayleigh number near $R a \approx 23,500$, where the Nusselt number departed from unity. The flow remained steady up to $R a=2,00,000$ and changed to periodic with further increase in $R a$. Rotating case is also validated with the afore-mentioned study [29] for $R a=9 \times 10^{5}$ at different rotation rates. Table 2 shows the $\langle N u\rangle$ comparison at various Rossby numbers, which are in excellent agreement.

\subsection{Grid independence study}

For checking grid independence, numerical runs are performed at four different mesh sizes. The simulations are carried out at $\operatorname{Ra}=5 \times 10^{4}, \operatorname{Pr}=7$ and $\operatorname{Ro}=0.1$. The grids are structured and non-uniform with refinement near the top and bottom surfaces and lateral wall. Table 3 shows the average Nusselt number at the bottom plate and kinetic energy $K E=\frac{1}{2} \int_{v}\left|\boldsymbol{U}_{\boldsymbol{R}}\right|^{2} d v$, for various grid sizes with corresponding minimum mesh size in the radial $\left(\Delta r_{\text {min }}\right)$ and axial direction $\left(\Delta z_{\min }\right)$. For unsteady periodic flows, the temporal averaged quantities $K E$ and $\langle N u\rangle$ are calculated by considering sufficient number of time periods after reaching a statistically steady state. The difference between the 


\section{RRBC}

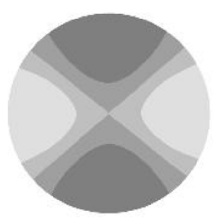

(a)

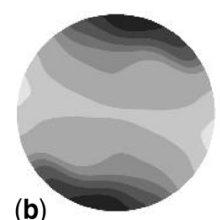

(b)
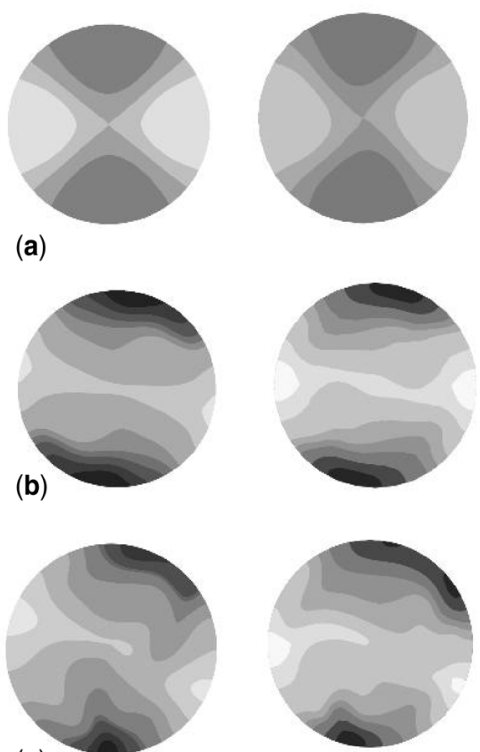

(c)
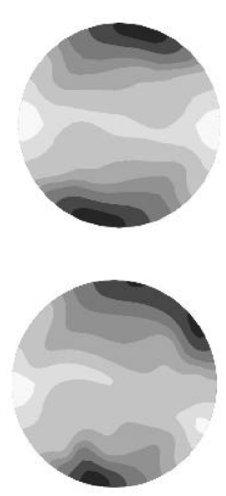

Figure 2. Mid-plane temperature deviation $\theta^{\prime}$ for different Rayleigh numbers. Dark (light) regions indicate $\theta^{\prime}<0\left(\theta^{\prime}>0\right)$ representing cold descending (warm ascending) fluid. Patterns on the right: Rüdiger and Knobloch [28] and left: present for (a) $R a=3600$, (b) $R a=30000$ and (c) $R a=50000$.

Table 1. Average Nusselt number at the hot wall for different $R a$ compared with those of Oresta et al [29].

\begin{tabular}{llcc}
\hline$R a$ & $\langle N u\rangle$ & $\langle N u\rangle$ Oresta et al $[29]$ & Error $(\%)$ \\
\hline 20000 & 1.0 & 1.0 & 0 \\
60000 & 2.46 & 2.54 & 3.15 \\
100000 & 3.34 & 3.37 & 3.83 \\
400000 & 6.45 & 6.50 & 0.77 \\
700000 & 8.65 & 8.35 & 3.6 \\
900000 & 9.48 & 9.03 & 4.8 \\
\hline
\end{tabular}

Table 2. Average Nusselt number at the hot wall for $R a=$ $9 \times 10^{5}$ at different $R o$ compared with those of Oresta et al [29].

\begin{tabular}{lccc}
\hline Ro & $\langle N u\rangle$ & $\langle N u\rangle$ Oresta et al [29] & Error (\%) \\
\hline 2 & 9.00 & 8.68 & 3.69 \\
0.2 & 4.95 & 5.20 & 4.81 \\
0.08 & 2.95 & 3.07 & 4.07 \\
0.056 & 2.02 & 2.08 & 2.98 \\
0.043 & 1.45 & 1.41 & 2.84 \\
0.036 & 1.13 & 1.19 & 5.04 \\
\hline
\end{tabular}

values computed at the finest and coarsest mesh is less than $1 \%$, which suggests a reasonable grid convergence. Considering the computational cost, accuracy and total

sampling time, the $83 \times 215 \times 83$ grid has been chosen. For all the simulations carried out in the present study, the boundary layers are aptly resolved by clustering more than 20 grid points within the thermal boundary layer.

\section{Results}

Numerical simulations are performed at four different Rayleigh numbers, namely $R a=3 \times 10^{4}, 5 \times 10^{4}, 7 \times$ $10^{4}$ and $10^{5}$, in a cylindrical container with aspect ratio $\Gamma=2$. Water $(\operatorname{Pr}=7)$ is considered as the working fluid. Rotation is applied along the vertical axis of the cylindrical domain. A wide range of rotation rate is considered, starting from non-rotating case to extremely high rotation rates. Rotation is expressed in terms of Rossby number $(R o=V / 2 \Omega H)$ and as $R o$ is inversely proportional to $\Omega, 1 /$ $R o$ directly measures rotation rate. The second-order explicit time marching algorithm uses a time increment $\Delta t=0.005$. Effect of rotation on heat transfer rate and stability of the system is investigated in the following subsections. Flow structures are studied by analysing the temperature iso-surfaces and vertical velocity contours at midvertical and mid-horizontal planes. Further, signals are extracted from nine different locations within the cylindrical domain to evaluate the unsteady (periodic) flow behaviour.

\subsection{Heat transfer analysis}

Area-averaged Nusselt number at the bottom plate $\langle N u\rangle$ is calculated for heat transfer analysis. Figure 3 shows $\langle N u\rangle$ at the bottom plate as a function of $1 / R o$ for different $R a$, where unfilled circles indicate that the solution obtained is periodic. For periodic cases, Nusselt number obtained is averaged over a specific time period of the respective flow. It can be clearly observed that heat transfer rate decreases steadily with increase in rotation rate (decrease in $R o$ ). For $R a=3 \times 10^{4}$, the average Nusselt number reduces with the rise in rotation rate and finally reaches a conduction-like stage with $\langle N u\rangle$ approaching unity. Similar observations are made for $R a=5 \times 10^{4}$ and $7 \times 10^{4}$. Here we can identify an analogy with flow and heat transfer across rotating cylinder, where the average Nusselt number decreases with the increase in rotation rate and increases with Reynolds number [30].

At $R a=10^{5}$, nearly $4 \%$ increase (from non-rotating case) is observed in $\langle N u\rangle$ at lower rotation rates $(R o \geq 10)$. With further increase in rotation rate, $\langle N u\rangle$ decreases steadily. This is in accordance with the observations made by Kunnen et al [20], Stevens et al [22] and Zhong et al [23] over a much higher $R a$ range. Rossby's experiments [16] with water showed that for $R a>10^{4}$ an increase in heat transfer rate of about $10 \%$ can be expected with the increase in rotation rates, with percentage of 
Table 3. Average Nusselt number at the bottom plate and total kinetic energy at different grid sizes.

\begin{tabular}{lcccc}
\hline Grid size & $67 \times 177 \times 67$ & $75 \times 195 \times 75$ & $83 \times 215 \times 83$ & $91 \times 236 \times 91$ \\
\hline$\Delta r_{\min }$ & 0.00676 & 0.00602 & 0.00542 & 0.00494 \\
$\Delta z_{\min }$ & 0.00686 & 0.00610 & 0.00549 & 0.00499 \\
$K E$ & 0.20225 & 0.20230 & 0.20231 & 0.20232 \\
$\langle N u\rangle$ & 2.76914 & 2.76959 & 2.75931 & 2.7590 \\
\hline
\end{tabular}

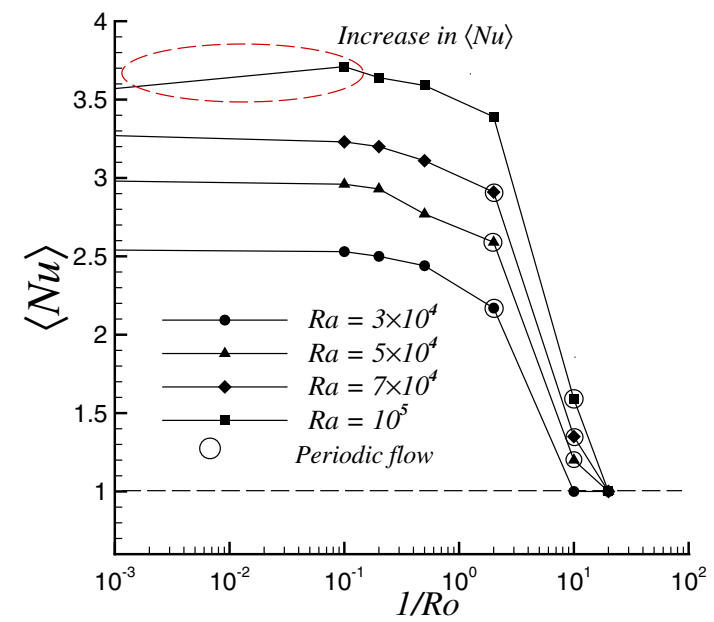

Figure 3. Variation of average Nusselt number with rotation rate for different $R a$.

increment dependent on the Rayleigh number. For lower $R a$ cases, we expect negligible increment in heat transfer rate at much lower rotation rates. In the present study we limit our $R o$ values to $\infty, 10,5,2,0.5,0.1$ and 0.05 .

Rotation primarily has a tendency to suppress the vertical motion, which leads to stratification of the flow along the rotation axis. Figure 4 shows the root mean square (r.m.s.) of vertical velocity and mean temperature variation along the axial direction for $R a=5 \times 10^{4}$ at different rotation rates. We calculate $w_{r m s}=\sqrt{\left\langle(w-\langle w\rangle)^{2}\right\rangle}$, where $\langle.$. indicates horizontal plane $(r-\phi)$ and time averaging. The magnitude of $w_{\text {rms }}$ increases near the bulk and decreases near the no-slip walls. The suppression of $w_{r m s}$ with increase in rotation is evident in this figure. At extremely high rotation rates $(R o \leq 0.1), w_{r m s}$ is considerably reduced, accompanied by stabilization along the vertical axis. At even higher rotation rates it can be expected that all the vertical velocity fluctuations are damped out and the magnitude of $w$ approaches zero, which is theoretically explained by the Taylor-Proudman theorem [4, 5]. The theorem states that in a homogeneous, inviscid flow dominated by Coriolis force, there is no variation of flow parameters along the flow axis, i.e., the flow behaves as 2-D in a plane orthogonal to the flow axis. The theorem is generally used in the context of geostrophic flows where the Coriolis force overshadows the other forces.
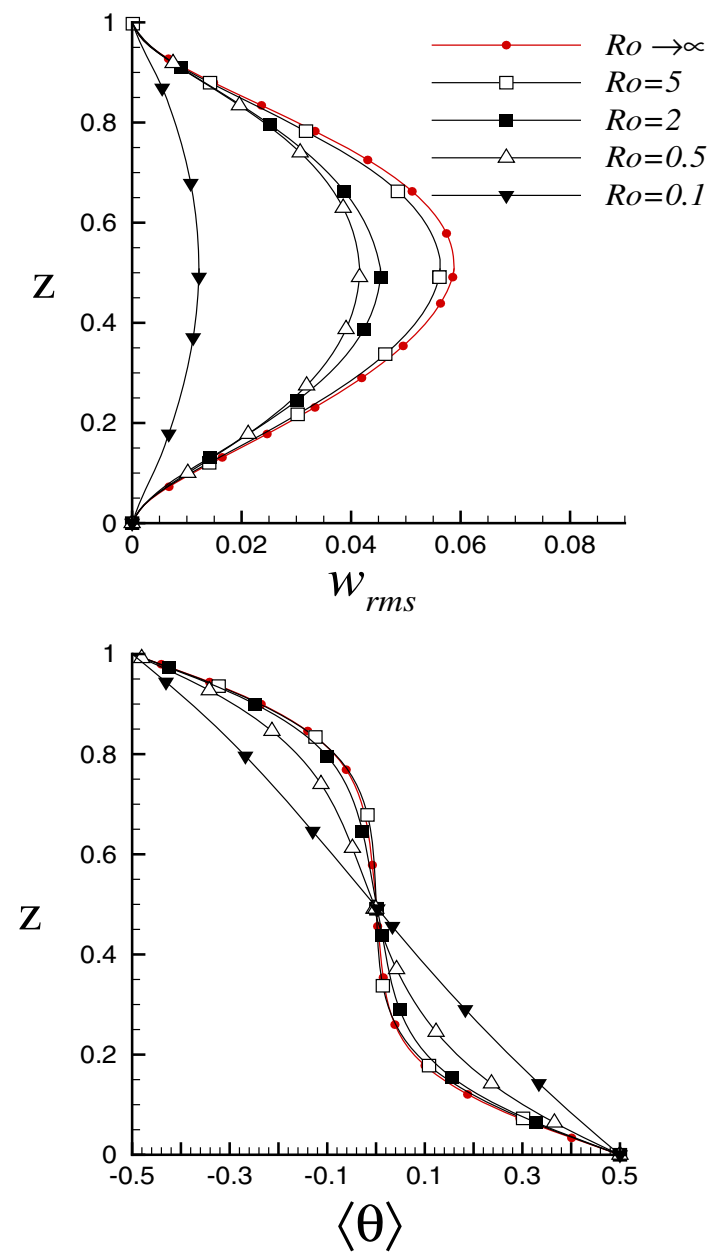

Figure 4. Variation (r.m.s.) of vertical velocity (top) and mean temperature (bottom) along the vertical direction for $R a=5 \times 10^{4}$ at different $R o$.

$$
\frac{\partial \boldsymbol{U}}{\partial z}=0
$$

Thus, there is no velocity variation along the vertical direction. Moreover, as we are dealing with a system with solid boundaries, $u_{z}=0$ (or $w$ ) on the boundaries implies

$$
\frac{\partial u_{r}}{\partial z}=0, \quad \frac{\partial u_{\phi}}{\partial z}=0, \quad u_{z}=0
$$

A similar trend is evident from the mean temperature profiles at different rotation rates. As the rotation rate 


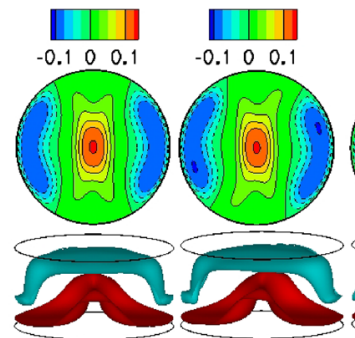

(a)

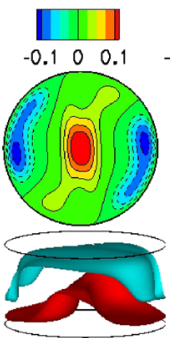

(c)

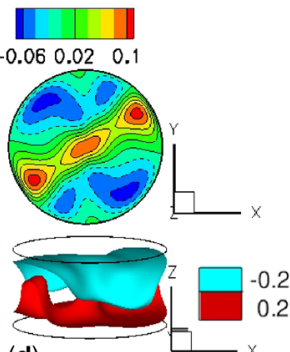

(d)

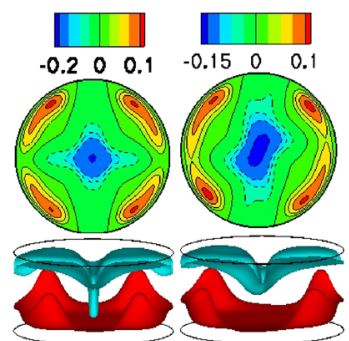

(a) (b)

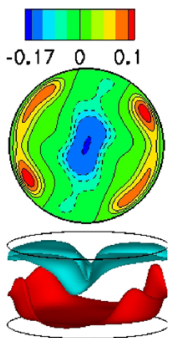

(c)

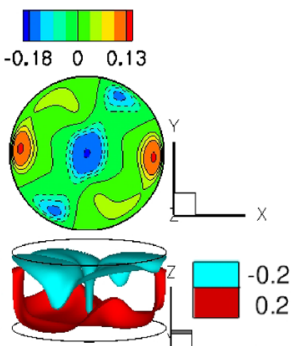

(d)

Figure 5. Upper plots are contours of vertical velocity at horizontal mid-plane with dotted (solid) lines indicating $w<0$ $(w>0)$. Lower plots are temperature iso-surfaces at $\theta=-0.2$ and 0.2 at $R a=3 \times 10^{4}$ for (a) $R o=\infty,(\mathbf{b}) R o=10$, (c) $R o=5$ and (d) $R o=2$.

increases, the gradient stabilizes along the vertical direction and the mean temperature profile approaches the linear one. At $R o \leq 0.1$ an almost linear profile is obtained, accompanied by $\langle N u\rangle$ approaching unity. This is a clear indication of suppression of the convective motion. As explained previously, at extremely high rotation rates the strong Coriolis force ceases the vertical motion and heat transfer occurs purely by conduction.

The drop in $\langle N u\rangle$ is accompanied by considerable changes in the flow structures. At all Rayleigh numbers, steady-state solutions are obtained at no-rotation or verylow-rotation rates. At lower rotation rates the flow patterns remain steady, which are similar to those of the non-rotating case. However, the degrees of skewness of the rolls increase with the increase in rotation as compared with straight rolls in the non-rotating case. In this region the drop in $\langle N u\rangle$ is also marginal, which is evident from figure 3.

The flow patterns obtained at $R a=3 \times 10^{4}$ for different Rossby numbers are shown in figure 5. For the non-rotating case, the vertical velocity contour at horizontal mid-plane $(z=0.5)$ and temperature iso-surfaces indicate that there exists a centrally ascending hot plume along with two descending cold plumes near the lateral wall. As rotation is applied, the plumes re-orient but the basic structure remains the same. However, at $R o=2$ the hot plume clearly breaks and a new flow pattern is obtained. Similar observations are made for $R a=5 \times 10^{4}$. At $R a=7 \times 10^{4}$, as shown in figure 6 , there exists a centrally falling cold plume and four rising hot plumes near the lateral wall for the non-rotating case. At low rotation rates $(\infty>R o \leq 5)$ this basic pattern remains the same, but an increase in skewness of the rolls with increase in rotation rate can be observed. With increase in rotation, two of the rising hot plumes diminish and the falling cold plume bifurcates. At $R o=2$ a considerable change in the flow structure can be observed. With further increase in the rotation rates, an unsteady periodic pattern is observed. The upward and downward roll patterns repeat after a specific interval of time. This is accompanied by a considerable drop in the heat transfer rate as observed in figure 3 . The patterns remained periodic

Figure 6. Upper plots are contours of vertical velocity at horizontal mid-plane with dotted (solid) lines indicating $w<0$ $(w>0)$. Lower plots are temperature iso-surfaces at $\theta=-0.2$ and 0.2 at $R a=7 \times 10^{4}$ for (a) $R o=\infty,(\mathbf{b}) R o=10,(\mathbf{c}) R o=5$ and (d) $R o=2$.

within a Rossby number range $0.5 \leq R o \leq 0.1$. Within this periodic regime, the intensity of the convective rolls drops with increase in the rotation rates. About 50\% drop in heat transfer is observed in this region.

On careful observation of the flow structures at low rotation rates $(R o \geq 5)$, it can be noticed that as the Rayleigh number is increased, number of plumes increases while they become localized. For $R a=3 \times 10^{4}$, shown in figure $5 \mathrm{a}-\mathrm{c}$, there exists a centrally ascending hot plume along with two descending cold plumes near the lateral wall. From the vertical velocity contours it is evident that the cold plumes are spread out along the lateral wall. However, for $R a=7 \times 10^{4}$ the plumes are more localized and four wall-bounded hot plumes dominate the region near the lateral wall.

\subsection{Periodicity and dual role of rotation on stability}

Rotational force tends to destabilize the flow that is otherwise steady. At moderate rotation rates, the flow destabilizes and becomes periodic. At $R o=0.1$ and 0.5 we obtain periodic flows, where the upward and downward plumes switch after a specific interval of time. However, extremely high rotation tends to stabilize the flow by inhibiting the convection rolls, as explained previously using the Taylor-Proudman theorem. The dual role of rotation on stability of RBC is evident here.

The time trace of vertical velocity obtained at mid-height close to the lateral wall $(r=0.9, \phi=0, z=0.5)$ for $R a=$ $5 \times 10^{4}$ and $R o=0.5$ is shown in figure 7 . The signal clearly depicts the periodic nature of the flow. The peak-to-peak distance of the signal is calculated to estimate time period $\left(T_{p}\right)$ of the flow. To study the flow dynamics, flow structures are analysed at five different time instants over $T_{p}$. Flow patterns corresponding to these time instants are shown in figure 9. The complete cycle of events are captured within a single time period, after which the flow repeats (see frames (a) and (e)). It can be clearly seen that the flow patterns observed at (c) and (d) are reverse of those at (a) and (b), 


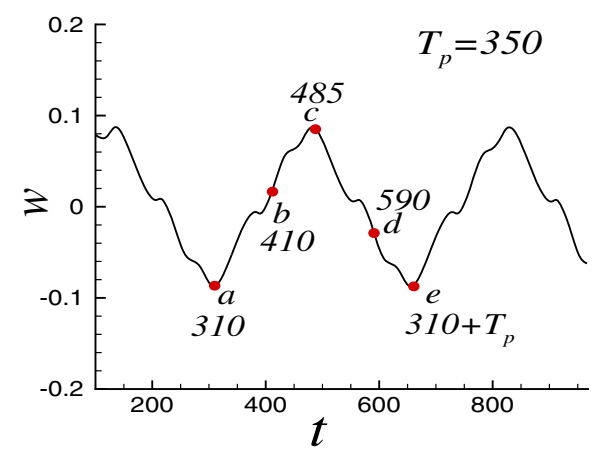

Figure 7. Time signal of vertical velocity at $r=0.9, \phi=0, z=$ 0.5 showing different time instants for $R a=5 \times 10^{4}$ and $R o=0.5$.

respectively. The regions where there is an upward flow in (a) (b) have downward flow in (c) (d) and vice-versa. The nearidentical flow patterns in frames (a) and (e) confirm the time periodicity of the flow. Periodic flows obtained at $R a=$ $5 \times 10^{4}$ and $10^{5}$ are shown in figures 9, 10 and 13. Flow structures obtained at $R a=7 \times 10^{4}$ follow a trend similar to those at $R a=5 \times 10^{4}$. As explained previously, the frames (a)-(e) in these figures represent the flow structures obtained at almost equispaced time instants within their corresponding flow time period.

To further substantiate the flow periodicity and switching, the skewness of vertical velocity fluctuations $S_{k}$ at midvertical plane is evaluated. Here $S_{k}=\left\langle\left(w^{\prime}\right)^{3}\right\rangle_{H} /\left\langle\left(w^{\prime}\right)^{2}\right\rangle_{H}^{3 / 2}$, where $w^{\prime}=w-\langle w\rangle_{H}$ and $\langle. .\rangle_{H}$ indicates horizontal plane $(r-\phi)$ averaging. The time evolutions of $S_{k}$ for $R a=$ $5 \times 10^{4}$ at $R o=0.5$ and 0.1 are shown in figure 8 . The signals switch from positive to negative at regular time intervals, suggesting periodic flow switching. Moreover, the time period of the $S_{k}$ signals is the same as that of the vertical velocity signals discussed before (see figure 7 for $R o=0.5)$. We observe an increment in the maximum skewness (peak of $S_{k}$ ) with the increase in rotation rate.

\subsection{Bulk mode and wall mode}

Mode transitions occur in RRBC due to the interplay between the buoyancy and Coriolis forces. In low rotation cases, the buoyancy force dominates over the Coriolis force and convection rolls can be seen all over the cylindrical domain. This is called bulk mode convection. For all Rayleigh numbers, bulk mode convection is observed at $R o \geq 0.5$. Figure 9 shows the bulk mode convection obtained for $R a=5 \times 10^{4}$ at a moderate rotation rate $R o=0.5$. It can be clearly seen that convection rolls scale almost the entire flow domain. As the rotation rate is increased, the Coriolis force overshadows the buoyancy force. As a result the buoyancy force becomes too small to sustain the convection rolls throughout the flow domain. The dominant Coriolis force pushes the convection rolls
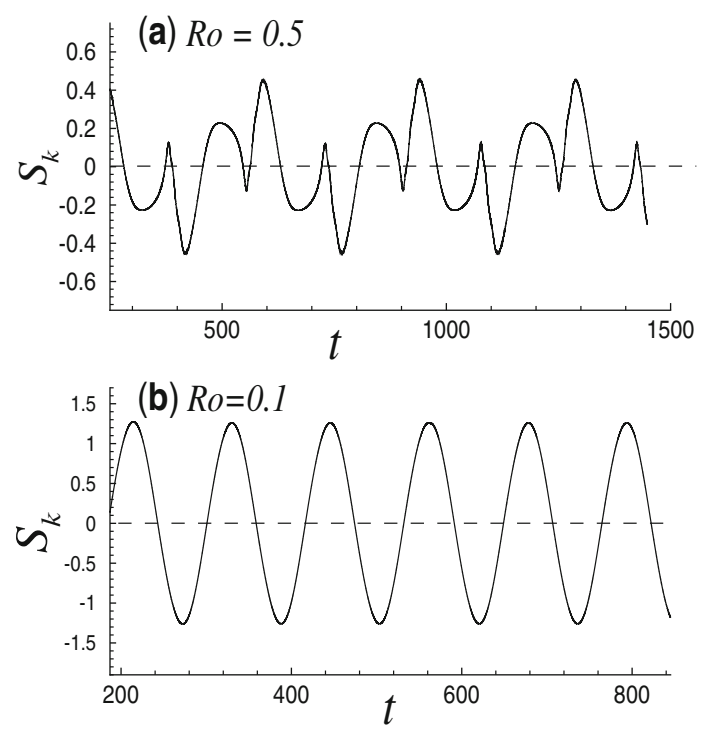

Figure 8. Time signal of skewness of vertical velocity at midvertical plane $(z=0.5)$ for $R a=5 \times 10^{4}$ at (a) $R o=0.5$ and (b) $R o=0.1$.

towards the lateral wall, thus resulting in a wall mode convection. In wall mode convection, pairs of wall-bounded hot plumes rise and cold plumes fall along the lateral walls. Figure 10 shows the wall mode convection obtained for $R a=5 \times 10^{4}$ at $R o=0.1$. The temperature iso-surfaces remain flatter in the bulk region and warp near the lateral wall. Vertical velocity contours indicate the presence of axial motion only near the walls. Wall modes can be considered as a preceding step before approaching towards a conduction-like profile. The suppression of flow due to rotation first occurs near the bulk and then moves towards the walls. Wall modes are associated with a drastic drop in the average $N u$. Further increase in the rotation rate yields a static conductive state with $\langle N u\rangle$ approaching unity.

To obtain further insights into the wall and bulk mode convection, temperature and vertical velocity at a vertical mid-plane are investigated. The changes along the axis of the cylinder can be observed evidently here. Figures 11 and 12 show the contours of temperature and vertical velocity at vertical mid-plane $(\phi=0)$ for $R a=7 \times 10^{4}$ at $R o=0.5$ and $R o=0.1$, respectively. Solid lines indicate positive values of temperature and vertical velocity while dotted lines correspond to negative values. At a moderate rotation of $R o=0.5$ the contours show that there exist a centrally rising hot plume along with two falling cold plumes on either side of the wall. As rotation is increased to $R o=0.1$, the temperature profile stabilizes near the bulk region (see figure 12) and wavers near the lateral wall. Stratification of the temperature profile can be clearly observed here. The vertical velocity contours show convection rolls only near the wall. More importantly, a uniform flow stabilization along the axis of the cylinder is evident.

At $R a=10^{5}$ and $R o=0.1$, although convection rolls are present mainly near the walls, unsteadiness can also be 


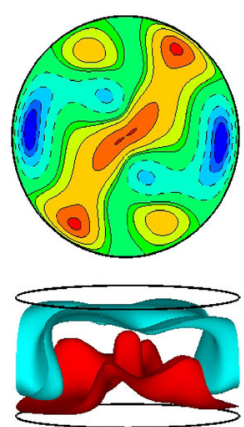

(a)

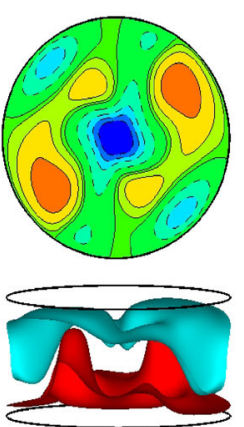

(b)

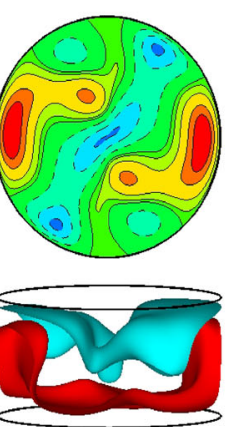

(c)

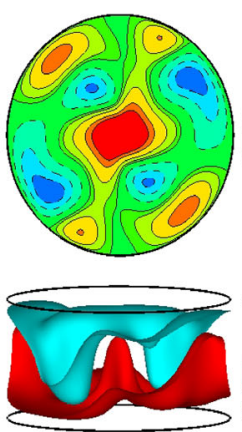

(d)

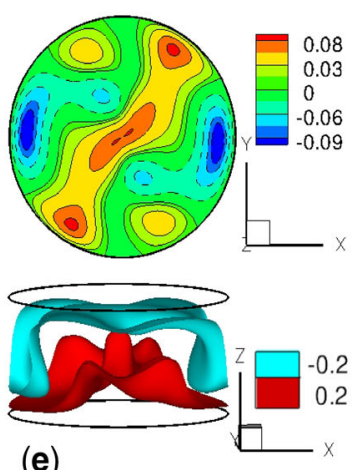

(e)

Figure 9. Upper plots are contours of vertical velocity at horizontal mid-plane with dotted (solid) lines indicating $w<0$ ( $w>0$ ). Lower plots are temperature iso-surfaces at $\theta=-0.2$ and 0.2 at $R a=5 \times 10^{4}, R o=0.5$ for (a) $t=310$, (b) $t=410$, (c) $t=485$, (d) $t=590$ and (e) $t=660$.

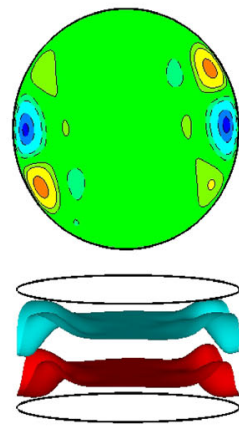

(a)

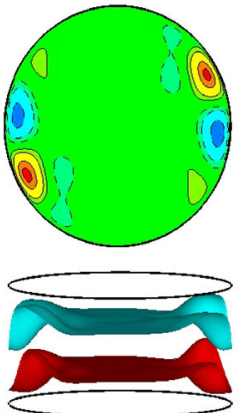

(b)

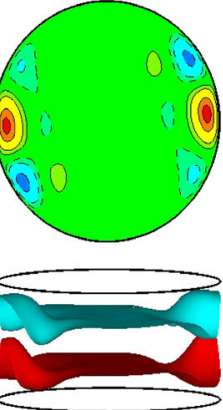

(c)

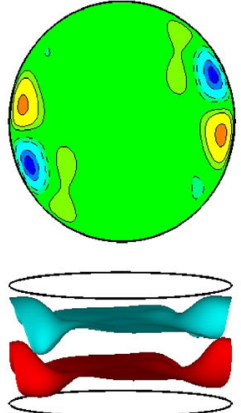

(d)

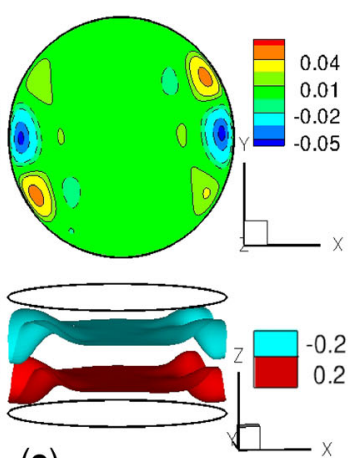

(e)

Figure 10. Upper plots are contours of vertical velocity at horizontal mid-plane with dotted (solid) lines indicating $w<0$ ( $w>0$ ). Lower plots are temperature iso-surfaces at $\theta=-0.2$ and 0.2 at $R a=5 \times 10^{4}, R o=0.1$ for (a) $t=290,(\mathbf{b}) t=315$, (c) $t=340,(\mathbf{d})$ $t=375$ and (e) $t=405$.

observed near the bulk region. The temperature iso-surfaces are not as flat as compared with the $R a=5 \times 10^{4}$ and $7 \times$ $10^{4}$ cases. Figure 13 shows that temperature iso-surfaces are wavered and the vertical velocity contours show axial motion near the bulk region. Thus, the flow cannot be categorized as pure wall mode. This is because at $R a=10^{5}$ the buoyancy force is much higher and a stronger rotational force is required to counter it. On closer observation of the temperature iso-surfaces it can be seen that at $R o=0.1$, with increase in Rayleigh number, flow stabilization near the bulk decreases. At $R a=5 \times 10^{4}$, temperature iso-surfaces are evidently flatter (see figure 10) near the bulk. However, at $R a=7 \times 10^{4}$ although it is flattened, a small amount of undulation can be seen. At $R a=10^{5}$, these undulations are triggered. This can also be substantiated by analysing the signals from different probes within the cylindrical domain, which is explained in the next section.

To connect the convection modes with heat transfer rate, we analyse the contours of Nusselt number at the bottom wall $(z=0)$ and near the mid-vertical plane $(z=0.5)$ for
$R a=5 \times 10^{4}$ at $R o=0.5$ and 0.1 (see figure 14). For bulk mode convection the maximum heat transfer rate is always near the bulk region, while it is concentrated near the lateral wall for wall mode convection. Further, the contours show that the Nusselt number distribution is more localized at $R o=0.1$ compared with $R o=0.5$. We observe about $50 \%$ reduction in $\langle N u\rangle$ as the flow transits from bulk mode to wall mode convection.

\subsection{Time evolution of the flow}

To analyse the characteristics of periodic flows, time signals at nine different points (shown in figure 15) are recorded. Periodicity of the flow, convection modes, mode transitions and possible flow reversals can be identified by analysing these time traces. The vertical velocity and temperature signals obtained from the afore-mentioned numerical probes at $R a=5 \times 10^{4}$ are shown in figures 16 and 17 for two different Rossby numbers $(R o=0.5$ and 0.1). Signals obtained from $S 7, S 8$ and $S 9$ probes are not 

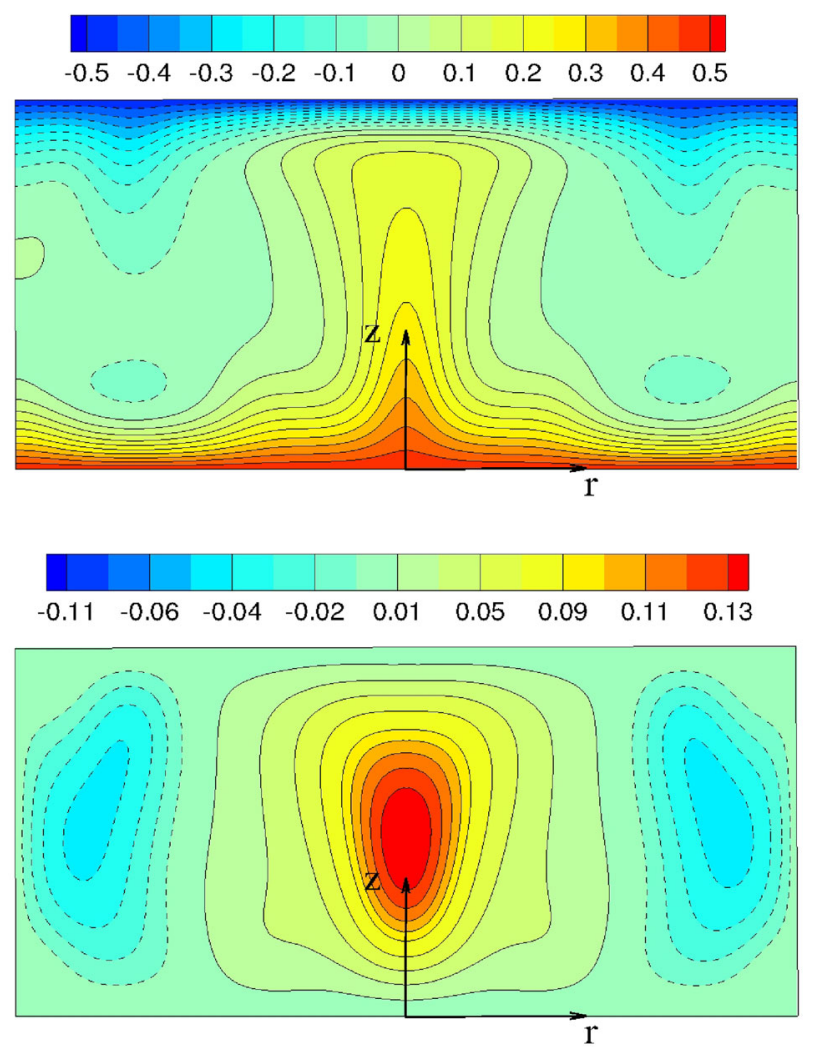

Figure 11. Contours of temperature (top) and vertical velocity (bottom) for $R a=7 \times 10^{4}$ and $R o=0.5$ at a vertical mid-plane, $\phi=0$.

shown here as they follow a trend similar to those of S1, S2 and S3, respectively. Periodic nature of the flow can be clearly recognized from these plots, where the signals at all points repeat after a specific interval of time. Further, the vertical velocity signals shift from positive to negative, indicating upward and downward flow switching. At $R o=$ 0.5 the vertical velocity signals on opposite sides of the lateral wall $\left(180^{\circ}\right.$ apart) exactly overlap with each other and the signal along the axis follows a trend that is inverse of these. However, at $R o=0.1$ no such trend is observed as the time traces along the bulk are considerably quiescent. The afore-mentioned rotation rates are the representatives of two different modes of convection. At $R o=0.5$, as shown in figure 16, along the mid-horizontal plane, the peak-to-peak amplitude of vertical velocity signals near the bulk is considerably higher than those near the walls. Further, the temperature signals at all points show ample unsteadiness, indicating the presence of convective motion throughout the flow domain. Consequently, as observed in the previous analyses, the flow at $R o=0.5$ can be categorized as bulk mode convection.

However, at $R o=0.1$ (see figure 17), temperature signals along the axis of the cylindrical domain are almost steady and those near the wall are unsteady. This indicates that temperature profile near the bulk has become
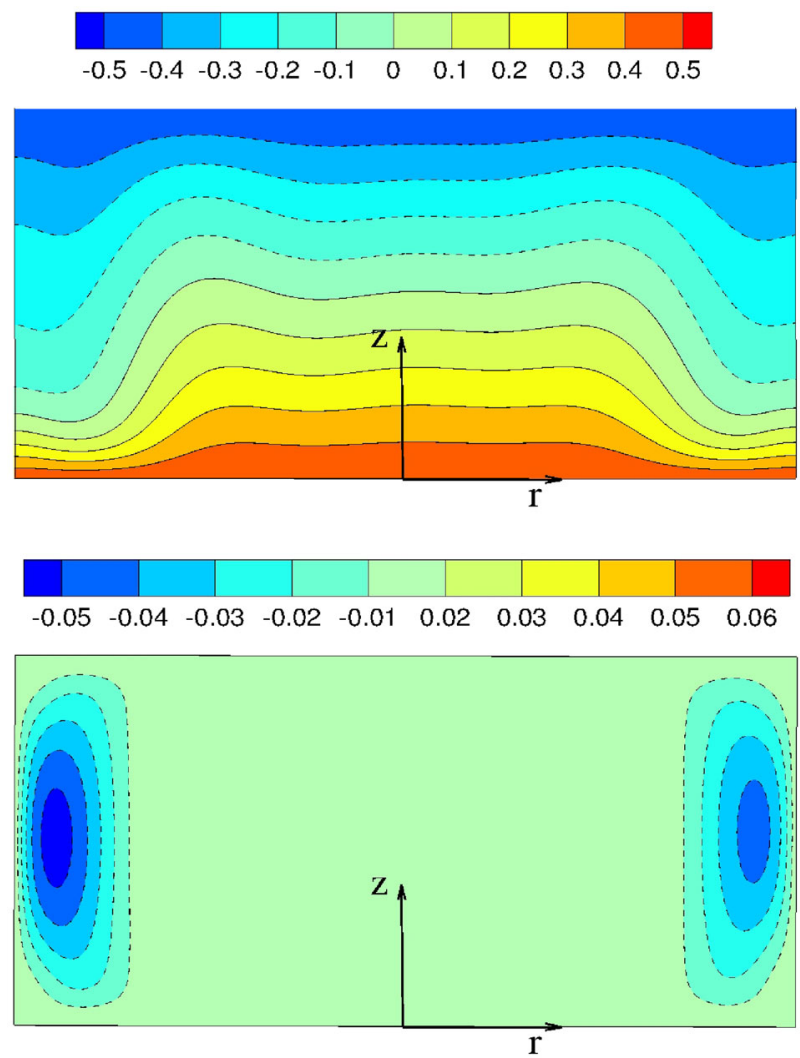

Figure 12. Contours of temperature (top) and vertical velocity (bottom) for $R a=7 \times 10^{4}$ and $R o=0.1$ at a vertical mid-plane, $\phi=0$.

stratified as strong rotation inhibits all the convection rolls. A similar observation can be made from the vertical velocity time traces, where the signals are clearly suppressed near the bulk. Along each horizontal line, vertical motion is detected only for signals near the wall. Along the axis of the cylindrical domain, the signals are broadly steady with magnitude approaching zero. This is a clear evidence of wall mode convection. The maximum peakto-peak amplitude of vertical velocity is always detected near the horizontal mid-plane in both the modes. For bulk mode convection it is near the bulk while for wall mode it is near the lateral walls. At $R o=0.5$ the time traces from all points are quasi-periodic and irregular while at $R o=$ 0.1 they are periodic and regular. Thus, a reduction in the frequency content of the signals can be observed with the increase in rotation rate, which is also a clear indication of flow stabilization. With further increase in rotation, higher harmonics are damped and flow finally attains a steady state.

To quantify the periodicity of the flow, Fourier transform of the temperature signals at $\mathrm{S} 1, \mathrm{~S} 2, \mathrm{~S} 4$ and $\mathrm{S} 5$ is calculated. The power spectral densities of these signals for $R a=5 \times 10^{4}$ at $R o=0.5$ and 0.1 are shown in figure 18 . It can be observed that the frequency content in the signals is considerably reduced with the increase in rotation rate. This 


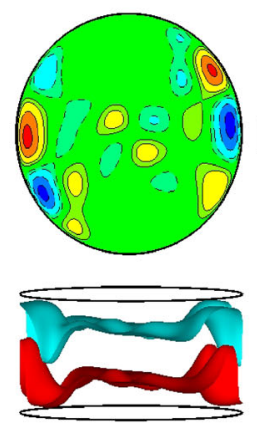

(a)

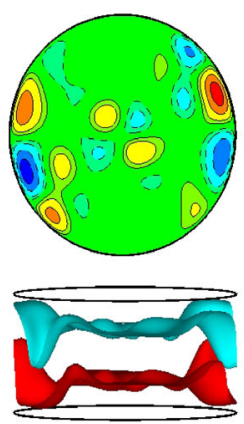

(b)

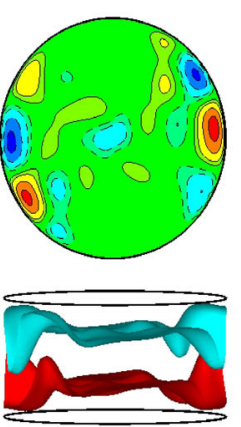

(c)

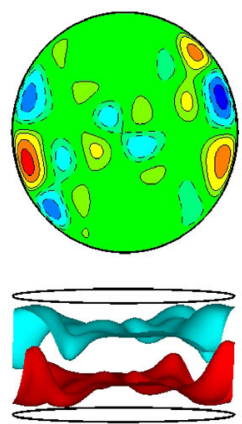

(d)
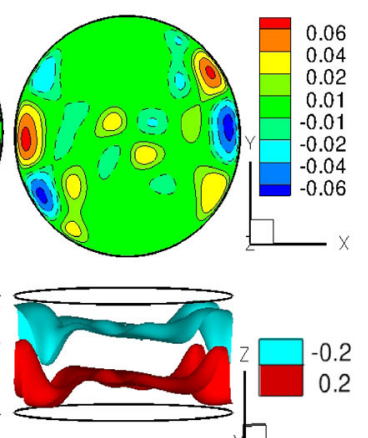

(e)

Figure 13. Upper plots are contours of vertical velocity at horizontal mid-plane with dotted (solid) lines indicating $w<0$ ( $w>0$ ). Lower plots are temperature iso-surfaces at $\theta=-0.2$ and 0.2 at $R a=10^{5}, R o=0.1$ for (a) $t=225$, (b) $t=260$, (c) $t=300$, (d) $t=340$, (e) $t=365$.

is accompanied by the reduction in the r.m.s. values (see table 4). The multiple peaks in the power spectrum for $R o=0.5$ indicate a quasi-periodic flow. For bulk mode convection $(R o=0.5)$ the signals near the bulk show maximum r.m.s. value. However, at $R o=0.1$, maximum r.m.s. is detected near the lateral wall and those near the bulk are close to zero. This clearly suggests the flow stabilization in the bulk region for wall mode convection.

As rotation rate is increased, time period of the periodic flows drops. Table 5 shows the time period $\left(T_{p}\right)$ at different $R a$ and $R o$ computed from the signals obtained from different numerical probes. It can be observed that $T_{p}$ drops considerably with increase in rotation. For $R a=5 \times 10^{4}$ at a moderate rotation rate of $R o=0.5$ the time period is 350 , while with the increase in rotation rate $(R o=0.1)$ it reduces to 115 . Similarly, for $R a=7 \times 10^{4}, T_{p}$ decreases (a) $R o=0.5(z=0.5)$

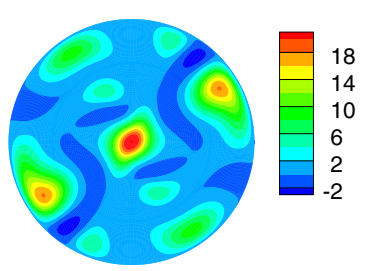

(b) $R o=0.5(z=0)$

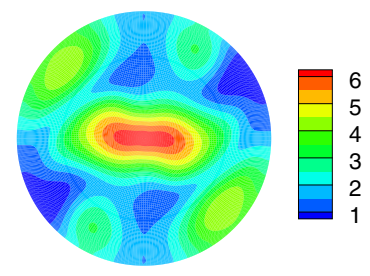

(c) $R o=0.1(z=0.5)$

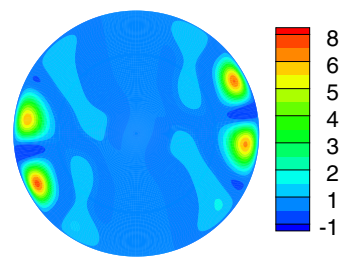

(d) $\operatorname{Ro}=0.1(z=0)$

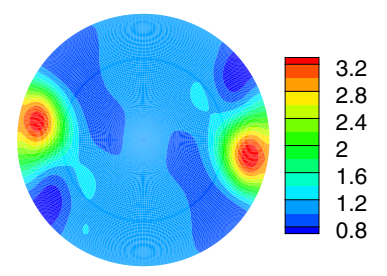

Figure 14. Contours of Nusselt number at (a) $R o=0.5$ and $z=0.5$, (b) $R o=0.5$ and $z=0$, (c) $R o=0.1$ and $z=0.5$ and (d) $R o=0.1$ and $z=0$ for $R a=5 \times 10^{4}$. from 400 to 125 as $R o$ is changed from 0.5 to 0.1 . Further, consistent drop in the peak-to-peak amplitude of vertical velocity signals substantiates the slow stabilization at higher rotation rates. For $R a=5 \times 10^{4}$ the maximum peakto-peak amplitude decreases from 0.32 to 0.1 as rotation rate increases from $R o=0.5$ to $R o=0.1$. A similar observation is made for $R a=7 \times 10^{4}$. With further increase in rotation rates $(R o=0.05)$ the amplitude diminishes, indicating no vertical motion, and a stratified temperature profile implies that heat transfer occurs purely due to conduction.

The time traces of vertical velocity and temperature for $R a=10^{5}$ at $R o=0.1$ are shown in figure 19. It can be clearly observed that although the maximum peak-to-peak amplitude of vertical velocity signals is detected near the walls, those along the axis also show considerable unsteadiness, indicating activity near the bulk region. Further, the temperature time traces near the bulk are

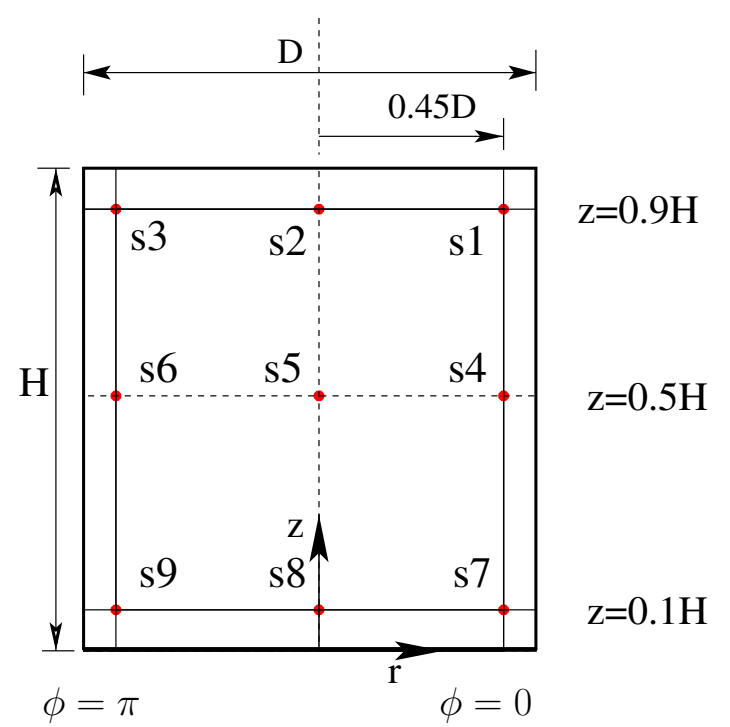

Figure 15. Mid-vertical planar view of the cylindrical domain showing locations of the numerical probes. 

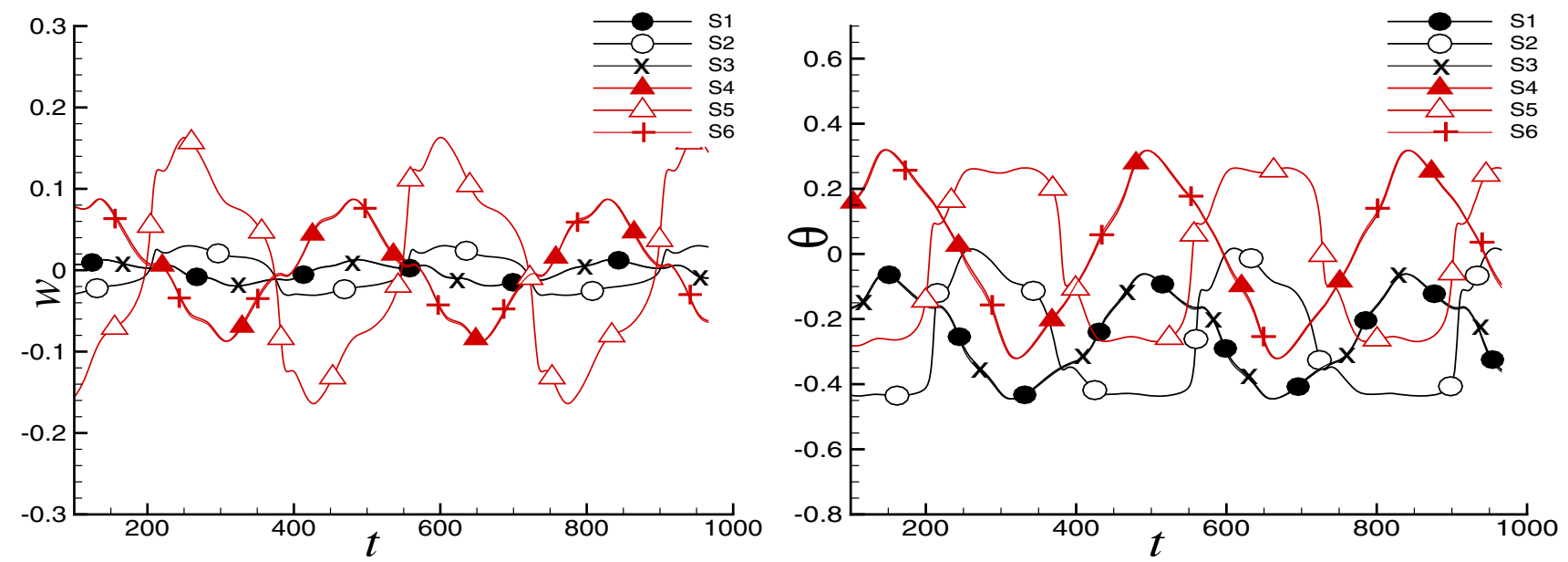

Figure 16. Temporal variation of vertical velocity (left) and temperature (right) for $R a=5 \times 10^{4}$ at $R o=0.5$.
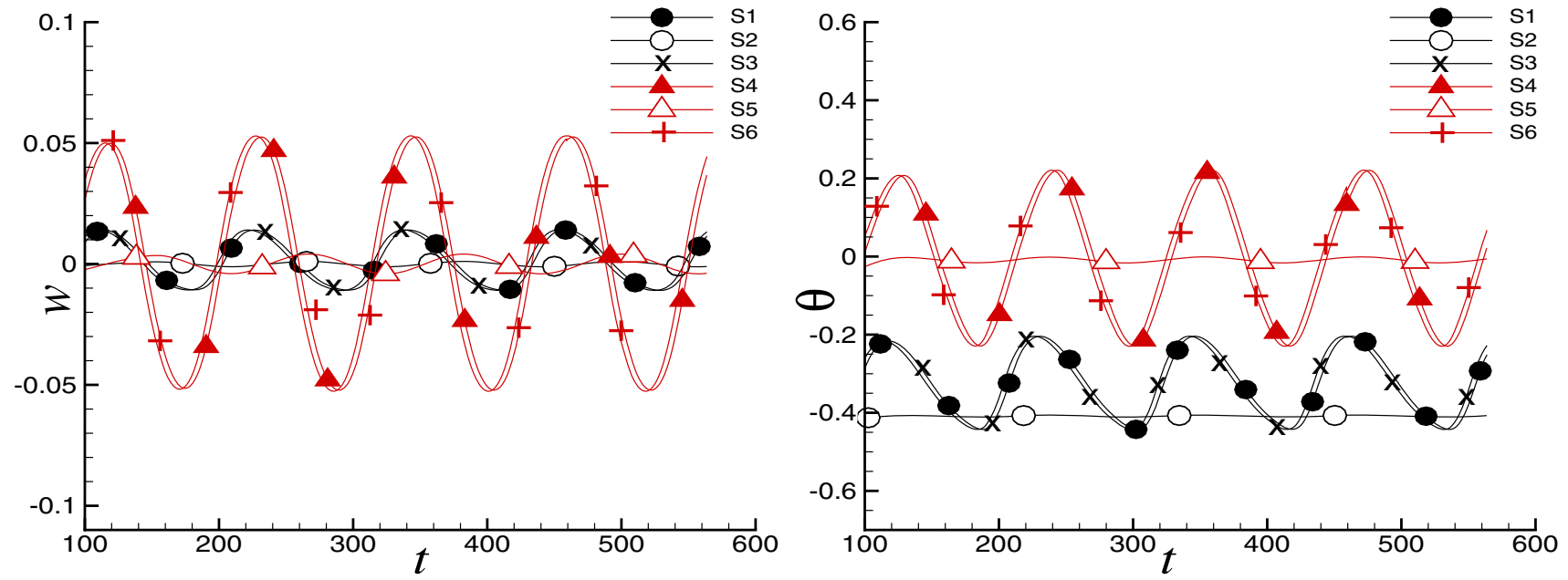

Figure 17. Temporal variation of vertical velocity (left) and temperature (right) for $R a=5 \times 10^{4}$ at $R o=0.1$.

Table 4. Root mean square value of temperature signals for $R a=5 \times 10^{4}$ at $R o=0.5$ and $R o=0.1$.

\begin{tabular}{lcc}
\hline Probe & $R o=0.5$ & $R o=0.1$ \\
\hline S1 & 0.125 & 0.084 \\
S2 & 0.173 & 0.002 \\
S4 & 0.199 & 0.155 \\
S5 & 0.224 & 0.005 \\
\hline
\end{tabular}

clearly unsteady. Thus, as explained in the previous section, flow cannot be categorized as pure wall mode here. This is due to the higher buoyancy force at $R a=10^{5}$, which sustains the convective motion even at a sufficiently higher rotation $(R o=0.1)$. With further increase in rotation rate it can be expected that this unsteadiness in the bulk region is damped and the system attains a pure wall mode convection.
Table 5. Time period of flow computed from recorded time traces.

\begin{tabular}{lcc}
\hline$R a$ & $R o$ & $T_{p}$ \\
\hline $3 \times 10^{4}$ & 0.5 & 290 \\
$5 \times 10^{4}$ & 0.5 & 350 \\
$5 \times 10^{4}$ & 0.1 & 115 \\
$7 \times 10^{4}$ & 0.5 & 400 \\
$7 \times 10^{4}$ & 0.1 & 125 \\
$10^{5}$ & 0.1 & 140
\end{tabular}

\section{Summary and conclusions}

A detailed numerical investigation on the effect of rotation for $\mathrm{RBC}$ in a cylindrical domain at low $R a$ regime is carried out in the present work. Though there are many existing 

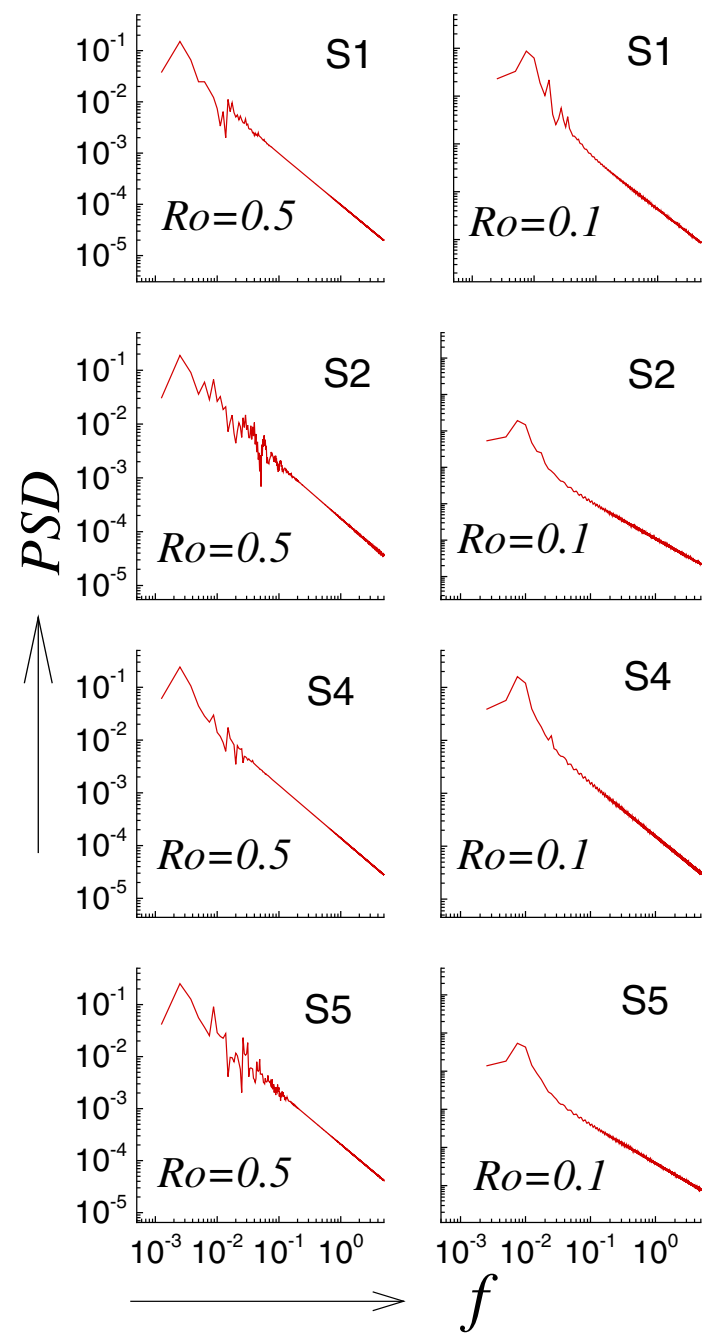

Figure 18. Power spectral density of the temperature signals from numerical probes S1, S2, S4 and S5 for $R a=5 \times 10^{4}$ at $R o=0.5$ (left panel) and 0.1 (right panel). works of literature on identifying the critical Rayleigh number for the onset of wall and bulk modes at constant rotation rates, the effect of rotation rate on convection mode transitions at a fixed $R a$ (at low $R a$ regime) is a less explored area. The present study investigates the bulk to wall mode transition with the increase in rotation rate and its relation with the Nusselt number at $R a \leq 10^{5}$.

The results show that rotation primarily has an inhibiting effect on the heat transfer rate. For $R a=3 \times 10^{4}, 5 \times 10^{4}$ and $7 \times 10^{4}$ the average Nusselt number at the bottom plate drops with the increase in rotation rate. The decline is meager at lower rotation rates and becomes significant at higher rotation rates. For $R a=10^{5}$ an increase in $\langle N u\rangle$ of about $4 \%$ is obtained at low rotation rates before it drops at higher rotation rates. Though this increase in heat transfer rate is not observed at lower Rayleigh numbers, we expect a similar trend in this region $\left(R a<10^{5}\right)$ at much higher Rossby numbers $(\infty>R o>10)$, which are not covered in the present study. Finally, a static conduction stage is reached, where the strong rotation force attenuates all the convection rolls, resulting in a stratified flow.

With the increase in rotation rate, different flow regimes are identified. The system remains steady at low rotation rates with the flow patterns similar to those in the nonrotating case. Within this regime, an increase in the skewness of convection rolls is observed with the increase in rotation rates. Thus the flow patterns slowly depart from that obtained at non-rotating case as the rotation rate increases. This is followed by a periodic regime at moderate rotation rates, where the time period of the flow decreases with the increase in rotation rate. At the onset of this periodic regime, bulk mode of convection is observed. Convection rolls can be seen all over the domain and the signals from all the numerical probes placed at various locations in the cylindrical domain show considerable
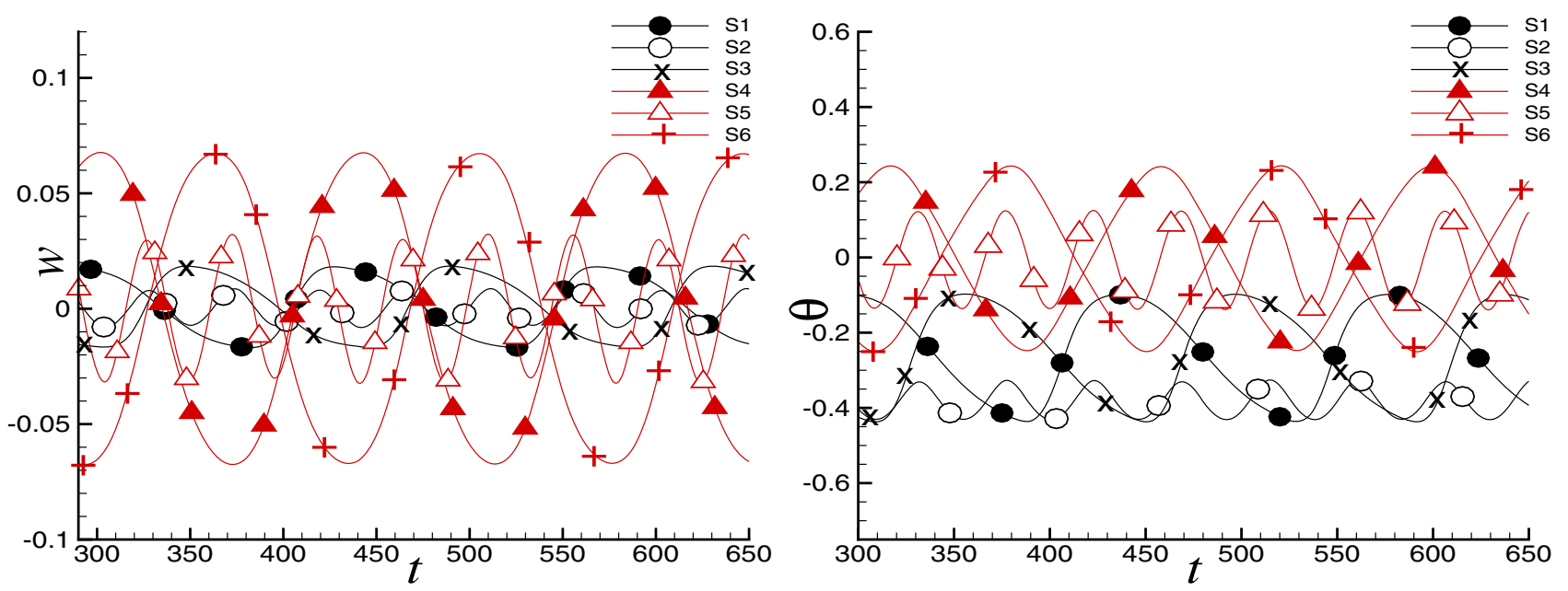

Figure 19. Temporal variation of vertical velocity (left) and temperature (right) for $R a=10^{5}$ at $R o=0.1$. 
variation. As the rotation rate is increased further, convection rolls become less pronounced accompanied by a drop in average $\langle N u\rangle$. With stronger rotation force, the flow stabilizes near the bulk and the convection rolls appear only near the lateral walls, which is supported by insignificant variations in the signals near the bulk. This regime is identified as wall mode convection, wherein $50 \%$ reduction in heat transfer rate is observed.

The flow is susceptible to unsteadiness at lower $R a$ than at higher $R a$ for the same rotation rate. Apparently, the dual role of rotation on the stability of RayleighBénard flows can be observed here. At moderate rotation rates, rotation destabilizes the flow, resulting in periodic flows. However, at high rotation rates, strong rotation force inhibits the convection rolls, resulting in flow stabilization.

\section{Acknowledgements}

All simulations have been carried out in the newly set up 'Param-Ishan' computing facility at the institute.

\section{Nomenclature}

$\alpha \quad$ thermal diffusivity

$\beta \quad$ thermal expansion coefficient

$v \quad$ kinematic viscosity

$\Gamma \quad$ aspect ratio

$\Omega \quad$ rotation rate

$\phi \quad$ azimuthal angle

$\theta \quad$ non-dimensional temperature

$\theta_{\text {con }} \quad$ conduction temperature profile

$\theta^{\prime}$ deviation from conduction profile

$\Delta T$ temperature difference

$D \quad$ cylinder diameter

$f$ frequency

$g \quad$ acceleration due to gravity

$H \quad$ cylinder height

$\mathrm{Nu} \quad$ Nusselt number

$\langle N u\rangle \quad$ average Nusselt number

$P \quad$ pressure

$P r \quad$ Prandtl number

$R \quad$ cylinder radius

$R a \quad$ Rayleigh number

Ro Rossby number

$S_{k} \quad$ skewness

Ta Taylor number

$T_{\text {。 }} \quad$ mean temperature

$T_{c} \quad$ temperature of cold plate

$T_{h} \quad$ temperature of hot plate

$T_{p} \quad$ flow time period

$\boldsymbol{U}_{\boldsymbol{R}} \quad$ velocity vector

$V \quad$ free-fall velocity

$w \quad$ vertical velocity component

$w_{\text {rms }} \quad$ vertical velocity (r.m.s.)

\section{References}

[1] Chandrasekhar S 1953 The instability of a layer of fluid heated below and subject to coriolis forces. Proc. R. Soc. Lond. A 217: 306-327

[2] Chandrasekhar S 1961 Hydrodynamic and hydromagnetic stability. Oxford University Press, Chapter 3, pp. 76-143

[3] Fultz D and Nakagawa Y 1955 Experiments on overstable thermal convection in mercury. Proc. R. Soc. Lond. A 235: 211-225

[4] Taylor G I 1923 Experiments on the motion of solid bodies in rotating fluids. Proc. R. Soc. Lond. A 104: 213-218

[5] Proudman J 1916 On the motion of solids in a liquid possessing vorticity. Proc. R. Soc. Lond. A 92: 408-424

[6] Veronis G 1959 Cellular convection with finite amplitude in a rotating fluid. J. Fluid Mech. 5: 401-435

[7] Buell J and Catton I 1983 Effect of rotation on the stability of a bounded cylindrical layer of fluid heated from below. Phys. Fluids 26: 892-896

[8] Ecke RE, Zhong F and Knobloch E 1992 Hopf bifurcation with broken reflection symmetry in rotating RayleighBénard convection. Europhys. Lett. 13: 177-182

[9] Goldstein HF, Knobloch E, Mercader I and Net M 1993 Convection in a rotating cylinder. Part 1. Linear theory for moderate Prandtl numbers. J. Fluid Mech. 248: 583-604

[10] Herrmann J and Busse F H 1993 Asymptotic theory of wallattached convection in a rotating fluid layer. J. Fluid Mech. 255: 183-194

[11] Kuo E Y and Cross M C 1993 Traveling-wave wall states in rotating Rayleigh-Bénard convection. Phys. Rev. E. 47: R2245-R2248

[12] Goldstein H F, Knobloch E, Mercader I and Net M 1994 Convection in a rotating cylinder. Part 2. Linear theory for low Prandtl numbers. J. Fluid Mech. 262: 293-324

[13] Zhang K, Liao X and Busse F H 2007 Asymptotic theory of inertial convection in a rotating cylinder. J. Fluid Mech. 575: 449-471

[14] Zhang K and Liao X 2009 The onset of convection in rotating circular cylinders with experimental boundary conditions. J. Fluid Mech. 622: 63-73

[15] Balasubramanian S and Ecke R E 2013 Experimental study of Rayleigh-Bénard convection in the presence of rotation. Int. J. Mater. Mech. Manuf. 1: 148-152

[16] Rossby H T 1969 A study of Bénard convection with and without rotation. J. Fluid Mech. 36: 309-335

[17] Zhong F, Ecke R and Steinberg V 1991 Rotating RayleighBénard convection: Kúppers-Lortz transition. Phys. D Nonlinear Phenom. 51: 596-607

[18] Ning L and Ecke R 1993 Rotating Rayleigh-Bénard convection: aspect-ratio dependence of the initial bifurcations. Phys. Rev. E 47: 3326-3333

[19] Marques F and Lopez J M 2008 Influence of wall modes on the onset of bulk convection in a rotating cylinder. Phys. Fluids 20: 024109

[20] Kunnen R P J, Geurtz B J and Clercx H J H, 2006 Direct numerical simulation of turbulent rotating Rayleigh-Bénard convection. In: Direct and large-eddy simulation VI, vol. 10, pp. 233-240 
[21] Stevens R J A M, Clercx H J H and Lohse D 2010 Optimal Prandtl number for heat transfer in rotating Rayleigh-Bénard convection. New J. Phys. 12: 359-364

[22] Stevens R J A M, Clercx H J H and Lohse D 2011 Numerical simulations of rotating Rayleigh-Bénard convection. In Direct and large-eddy simulation VIII, vol. 15, pp. 359-364

[23] Zhong J Q, Stevens R J A M, Clercx H J H, Verzicco R, Lohse D and Ahlers G 2009 Prandtl-, Rayleigh-, and Rossbynumber dependence of heat transport in turbulent rotating Rayleigh-Bénard convection. Phys. Rev. Lett. 102: 044502:1-4

[24] Kunnen R P J, Geurtz B J and Clercx H J H 2008 Breakdown of large-scale circulation in turbulent rotating convection. Europhys. Lett. 84: 24001:1-6
[25] Kunnen R P J, Geurtz B J and Clercx H J H 2010 Experimental and numerical investigation of turbulent convection in a rotating cylinder. J. Fluid Mech. 642: 445-476

[26] Peter S and De A K 2016 Wake instability modes for forced transverse oscillation of a sphere. Ocean Eng. 115: 48-59

[27] Ferziger J H and Peric M 2002 Computational methods for fluid dynamics. Springer, Chapter 5, pp. 97-116

[28] Rudiger S and Knobloch E 2003 Mode interaction in rotating Rayleigh-Bénard convection. Fluid Dyn. Res. 33: 477-492

[29] Oresta P, Stringano G and Verzicco R 2007 Transitional regimes and rotation effects in Rayleigh-Bénard convection in a slender cylindrical cell. Eur. J. Mech. B Fluids 26: 1-14

[30] Paramane S B and Sharma A 2010 Heat and fluid flow across a rotating cylinder dissipating uniform heat flux in 2D laminar flow regime. Int. J. Heat Mass Transf. 53: 4672-4683 